\title{
Simplified Damper Cage Circuital Model and Fast Analytical-Numerical approach for the analysis of Synchronous Generators
}

\author{
Stefano Nuzzo, Paolo Bolognesi, Chris Gerada and Michael Galea
}

\begin{abstract}
The long and enduring history of utilization of the wound-field synchronous generator in a large number of applications makes it one of the most known and consolidated electrical machine technologies. Thus its design, modelling and analysis processes have been widely exploited and implemented through various and different methods, including the equivalent circuit approach. When a damper cage is embedded within the rotor of the synchronous generator, its theoretical analysis becomes quite complicated. Thus, today numerical tools are being used. In this paper, an alternative way of modelling symmetric damper cages of salient-pole synchronous generators is presented. The proposed approach is embedded in the circuital model of the generator. A hybrid analytical-numerical model is implemented, permitting to accurately predict the voltage waveforms of the generator with excellent accuracy, however at a lower cost of computational resources than the pure numerical method. A case study of an off-the-shelf 400kVA machine is considered to develop and validate the proposed technique. The results are compared to corresponding finite-element and experimental evaluations for validation purposes.
\end{abstract}

Index Terms-Analytical Modelling, Damper Windings, Equivalent Circuit, Permeability Function, Winding Functions, Wound Field Synchronous Generators.

\section{INTRODUCTION}

$\mathrm{T}$ HE ever-more stringent power quality and efficiency requirements [1] and the advances in the computing resources, modelling tools and new materials [2] are leading to game-changing renovations of the classical design topologies and system-level architectures typical of woundfield synchronous generators (SGs)and their excitation systems [3].The need of this revamping process is also pushed by new and ever more challenging applications [4] where objectives such as costs, reliability and controllability make permanent magnet excitation systems an unsuitable solution.

Of all the design features that fall within this renovation process, particular focus has recently been given to the damper cage usually embedded inside the rotor of salient-pole SGs. During any operating condition, parasitic phenomena are

Manuscript received June 05, 2018; revised July 27, 2018; accepted Month $\mathrm{x}, \mathrm{xxxx}$.

S. Nuzzo, M. Galea and C. Gerada are with the Power Electronics, Machines and Control Group, University of Nottingham, Nottingham, UK (e-mail: stefano.nuzzo@nottingham.ac.uk) . induced in such a winding. At no-load, due to the tooth ripple, parasitic harmonics are present in the airgap flux density [5]. These have a major effect in machines featuring an open slot structure [6]. Additional parasitic effects are produced at onload operation by the armature reaction magneto-motive force. As a consequence, the bar currents can be high and the ensuing ohmic losses can deteriorate the machine efficiency. An overview of the importance and behaviour of damper windings in salient-pole SGs is provided in [6] and [7].

A number of recent publications dedicated to damper cage modelling and development can be found in literature. In [8] and [9], permeance models have been presented and implemented with the aim of accurately predicting the bar currents, even under normal operating conditions, since their effects are often less negligible than traditionally assumed. An innovative numerical integration method is proposed in [5].The more and more powerful tools of numerical analysis available today are capable of accurately predicting the performance of SGs considering the damper cage effects [2],[5], [6], [8]-[11]. In [12], a sensitivity study aimed at understanding the damper cage behaviour under steady state and transient conditions has been carried out. Investigations have been recently performed to understand the impact that this winding has on the quality of the output voltage waveforms [6], [11], [13]. In particular, the works presented in [6] and [11] have shown how it is possible to improve the voltage total harmonic distortion (THD) by tailoring the position of each bar, while trying to minimise the associated losses.

All the above has shown that significant improvement for synchronous generators, as requested by the changing general industrial requirements can be achieved by addressing the longforgotten damper cage winding. However, the nature of this winding makes it very difficult to analyse correctly and this also implies the need for powerful and accurate modelling techniques. While today these tools do exist, there is also a stringent need for more efficient tools and modelling techniques that can address not only accuracy but also the cost of computational resources.

In [14], such an efficient tool, coined as "hybrid analyticalnumerical modelling approach" was proposed. In this work, main focus was devoted to the damper cage, where it was shown that by setting certain assumptions related to its symmetric

M. Galea is also with the School of Aerospace, Faculty of Science and Engineering, University of Nottingham Ningbo, Ningbo, China (email: michael.galea@nottingham.edu.cn).

P. Bolognesi is with the Electrical Machines, Power Electronics and Drives Group, University of Pisa, Pisa, Italy (e-mail: p.bolognesi@ieee.org) 
layout, then an advanced, alternative model of the damper cage winding can be developed. Implementing this simplified damper cage model allows for a drastic reduction of the number of electromagnetic equations to be considered, when one considers the characterisation and analysis of the whole SG at no-load operation. In order to validate this technique against a practical case, the whole concept was applied to an off-the-shelf $400 \mathrm{kVA}$ alternator.

This paper thus takes the work firstly proposed in [14] and projects it to higher levels of theoretical and technical depth. This is achieved by considering other important aspects such as 1) the effects of the salient poles' profiles on the geometrical functions characterizing the SG, 2) an enhanced explanation of the damper cage winding functions (WFs) resulting from the relevant assumptions, 3) an improved investigation of the final machine inductances, 4) a full experimental validation of the presented concepts and 5) an explicative summary of the major advantages of the proposed model against the state-of-the-art.

As mentioned above, an extended regime of validation against purely numerical results and against experimental results is included in this paper. Focus is given to constantspeed no-load operation, as most SG manufacturers consider the voltage waveforms obtained in such conditions to be very significant to assess the power quality of their product. However, to highlight the flexibility of the hybrid model, onload predictions are also shown in the paper. This work thus highlights the extremely successful implementation of the proposed model.

\section{THE ANALYTICAL MODEL}

The basic structure of the proposed analytical model is presented in [15], where focus was given only to the purely conceptual and theoretical aspects. No practical examples were presented in [15], thus this paper proposes to investigate the model on a classical, wound-field SG, with special focus on the damper cage, which was fully overlooked in [15]. Due to the complexity of the phenomena associated to this winding and to its impact on the voltage waveform quality, incremental improvements on the basic model are necessary. First, the model is recalled in its general framework. Variations and updates are then described. All the numerical quantities and graphical examples reported in this paper will refer to a 3-phase alternator, whose 2-D half cross-section is shown in Fig. 1, where the major geometrical features can be observed. The rated apparent power and output voltage are $400 \mathrm{kVA}$ and $400 \mathrm{~V}$ respectively. In each salient pole, 6 aluminium bars are symmetrically embedded in the same positions around the polar axes. The end bar connections consist of aluminium pressplates, which reproduce the laminations' shape but with a larger thickness.

\section{A. Model Introduction}

The considered analytical model has been fully described in [15] and the main assumptions discussed in detail in [14]. However, in order to set the context, it is important to recall the hypotheses which are most relevant for the objectives of this work. These include

1) The separation of the phenomena involving the active sides of the windings from those occurring in the end windings;

2) negligible influence of leakage flux tubes on the behaviour of the main flux tubes (those responsible of electromechanical transformation), i.e. negligible cross saturation;

3) the 2-D approximation of the field map;

4) the magnetic linearity of the materials used for the stator and rotor cores, meaning that the dependency on the currents is neglected;

5) the constant value of the magnetic field intensity in the airgap with respect to the radial direction.

The above assumptions as well as the basic general framework of the model proposed in [15] are maintained for the analysis of the considered machine. However, it is clear that some updates are needed to achieve the main goal of this paper, i.e. estimating the SG voltage waveforms.

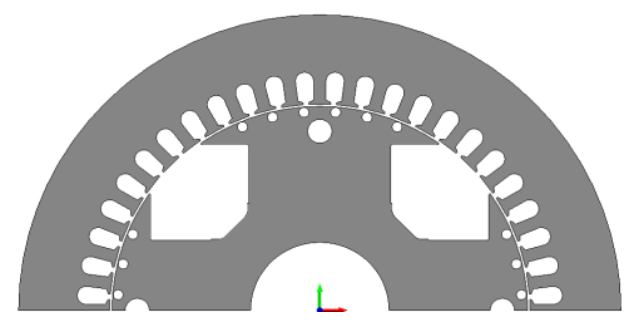

Fig. 1. 2-D half view of the core of the.SG considered as case study.

\section{B. Model Description}

Having introduced the general assumptions of the analytical model as in Section II.A and considering that these match the hypotheses of the analysis developed in [15], the expression of the matrix inductance function $\mathbf{L}(\alpha)$ given in (1) can be derived. This expression, whose accuracy has been confirmed by a number of works [3], [6], can be used to analyse the phenomena referred only to the flux tubes which contribute to the electromechanical energy transformation. It can be clearly seen that $\mathbf{L}(\alpha)$ plays a key role in the voltage vector equation shown in (2).Here the hypothesis of linear characteristics for all of the magnetic materials is assumed, as anticipated in Section II.A.

$$
\begin{aligned}
& \mathbf{L}(\alpha)=l \cdot \int_{0}^{1} \mu_{E}(\lambda, \alpha) \cdot \overline{\mathbf{N}}_{\mathbf{E}}(\lambda, \alpha) \cdot \overline{\mathbf{N}}_{\mathbf{E}}^{\mathbf{T}}(\lambda, \alpha) \cdot d \lambda \\
& \overline{\mathbf{v}}(t)=\mathbf{R} \cdot \overline{\mathbf{i}}(t)+\overline{\mathbf{M}}(\alpha(t), \overline{\mathbf{i}}(t)) \cdot \frac{d \alpha(t)}{d t}+\mathbf{L}(\alpha(t)) \cdot \frac{d \overline{\mathbf{i}}(t)}{d t}
\end{aligned}
$$

In (2), $\alpha$ is the angular position of rotor vs. stator, the vector $\overline{\mathbf{i}}$ is composed by the currents entering the machine phases (in broad sense, i.e. also including rotor circuits), $\mathbf{R}$ is the matrix containing the phase resistances and $\overline{\mathbf{M}}(\alpha, \bar{i})$ is the motional coefficients vector, which is obtained by partial derivation of the linked flux vector with respect to the variable $\alpha$, assuming currents as constants.

By examining (1) more in detail, it can be observed that $\mathbf{L}(\alpha)$ depends only on two functions. These are the equivalent permeability function $\mu_{E}(\lambda, \alpha)$ and the vector $\overline{\mathbf{N}}_{\mathrm{E}}(\lambda, \alpha)$ containing the equivalent winding functions (EWFs) of the machine. Always referring to (1), $l$ is the axial length of the machine cores and $\lambda$ is the normalized coordinate mapping the whole airgap 
along the tangential direction by spanning in the $[0,1)$ interval. For the sake of clarity, the expressions of $\mu_{E}(\lambda, \alpha)$ and $\overline{\mathbf{N}}_{\mathrm{E}}(\lambda, \alpha)$ are also provided in (3) and (4), respectively.

$$
\begin{aligned}
& \mu_{E}(\lambda, \alpha)=\mu_{0} \cdot \frac{2 \cdot \pi \cdot r_{G}(\lambda, \alpha)}{\varepsilon_{G}(\lambda, \alpha)} \\
& \overline{\mathbf{N}}_{\mathbf{E}}(\lambda, \alpha)=\overline{\mathbf{N}}(\lambda, \alpha)-\int_{0}^{1}\left(\frac{\mu_{E}(\lambda, \alpha)}{\int_{0}^{1} \mu_{E}(\lambda, \alpha) \cdot d \lambda}\right) \cdot \overline{\mathbf{N}}(\lambda, \alpha) d \lambda
\end{aligned}
$$

From (3), it is easy to infer that the equivalent permeability function contains all the information regarding the anisotropies featured by the specific device under analysis. In fact, in (3) $r_{G}(\lambda, \alpha)$ is the distance from the geometrical axis of the mean surface inside the airgap (i.e. the airgap mean radius) and $\varepsilon_{G}(\lambda, \alpha)$ represents the equivalent length of the field lines in the main airgap (i.e. the equivalent thickness of the airgap).

In (4), the vector $\overline{\mathbf{N}}(\lambda, \alpha)$ groups the WFs describing all the machine windings. Each element of such a vector contains the information related to a)the distribution of the conductors among the different groups (slots) and phases, b)their location along the tangential direction and c)their orientation along the axial direction according to the reference direction assumed for the currents entering the phases.

Having recalled the basic quantities of the proposed analytical model, it becomes possible to introduce new assumptions with the aim of accurately predicting the output voltage shape. First, the slot opening effects are treated. It is then discussed the way how the salient poles' profiles are implemented. The inherent implications on the function $\mu_{E}(\lambda, \alpha)$ are also detailed. On the other hand, given that the rotor bars are embedded in closed slots inside the rotor poles, these are considered negligible on the variation of the functions involved in (3). Even if the slots are closed, an equivalent slot opening could be considered [16] to model local saturation phenomena potentially occurring in correspondence of the iron bridges close to the damping bars. The exact location, width and shape of such saturated areas can vary from bar to bar depending on load levels and rotor position. Their inclusion would thus significantly complicate the computation of $\mu_{E}(\lambda, \alpha)$, going against the objective of reducing the model complexity and computational cost.

\section{The slot opening}

The stator slot openings and the related harmonics play a role of paramount importance on the actual waveform of the output voltage. In [15], these aspects were overlooked and therefore their inclusion in the model represents the first incremental improvement needed for the determination of the machine inductance matrix. As a first approximation, their equivalent profile can be represented with a trapezoidal shape as in Fig. 2 (in red), where also the original profile (in black) of the inner radius of the stator core vs. angular location is sketched. The slot opening width $c$ is assumed to be the same as in the real laminations, whereas the depth $a$ and the width $b$ of the ramp are considered as characteristic parameters. The values of the equivalent parameters characterizing the slots are intended to be determined through a tuning procedure, carried out by comparing the results with those obtained via a corresponding 2-D electromagnetic FE analysis of the SG.

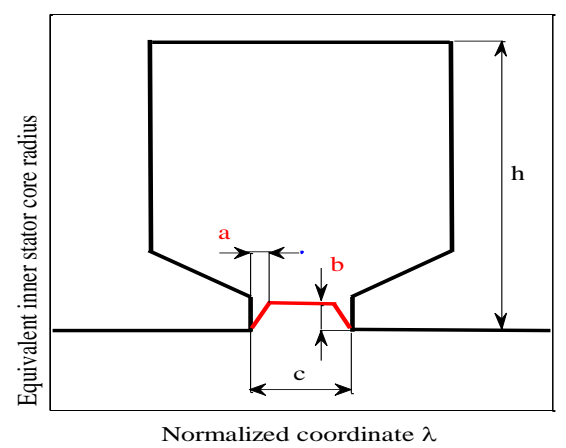

Fig. 2. The actual (simplified) equivalent inner stator profile.

\section{The salient poles}

Besides the slot openings, in salient-pole SGs it is very important to model the salient poles' profiles to achieve an accurate estimation of the main phenomena occurring in the electrical machine, especially those involving the voltage shape. Also these aspects were neglected in [15], although the general guidelines were provided. Therefore, their effects are included in the model by considering an equivalent profile as similarly done for the stator slot openings. In this case, a double trapezoidal variation of the outer radius of the rotor core vs. angular location is used to schematize the salient poles. This concept is shown in Fig. 3, where the distance $d$ between two adjacent poles is assumed to be the same as in the actual machine, while widths and heights of the ramps ( $e, f, g$ and $i$ ) are considered as characteristic parameters. As well as for the slot openings, the values of the characteristic parameters $e, f, g$ and $i$ are intended to be determined via a tuning procedure against the FE analysis.

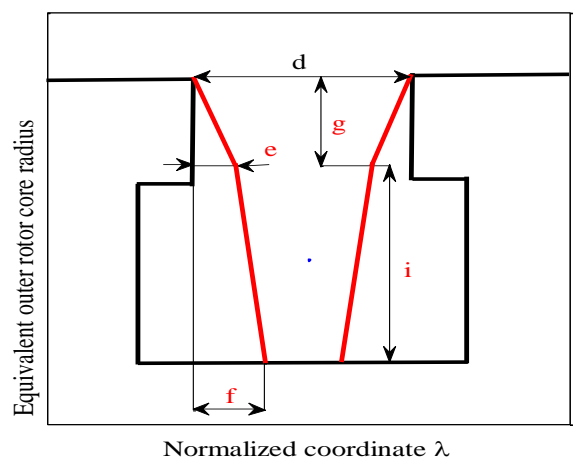

Fig. 3. The actual (simplified) equivalent outer rotor profile.

\section{$E$. The equivalent permeability function}

The implementation of the geometrical quantities involved in (3) through the procedures detailed in the previous sections allows to determine $\mu_{E}(\lambda, \alpha)$. This equivalent function plays a key role in (1) and the accuracy of its shape is thus essential to calculate the machine inductance matrix. The function $\mu_{E}(\lambda, \alpha)$ is shown in Fig. 4 at a specific, fixed rotor position. As salientpole SGs exhibit a symmetry that inherently results in a repetitive trend with period equal to $1 /(2 * p)$ of all the quantities involved in (3), $\mu_{E}(\lambda, \alpha)$ is plotted vs. $\lambda$ only for one repetition 
period. In Fig. 4, the effects of the slot openings and the rotor poles' profiles can be observed on such a function.

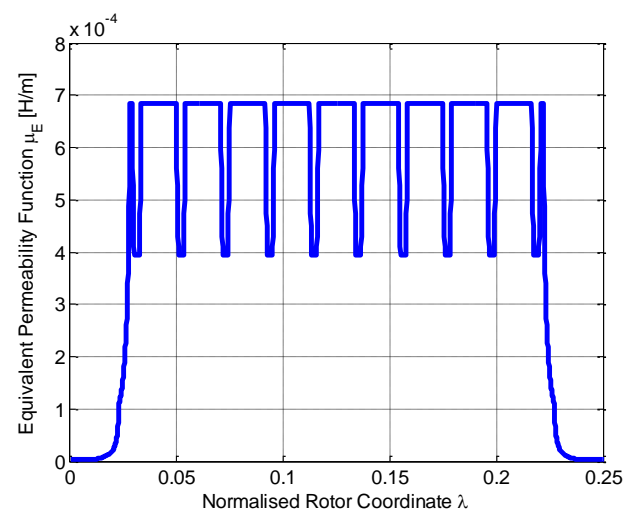

Fig. 4. Equivalent permeability function of the considered case study.

\section{F. Parameters tuning and methodology flowchart}

In sections II.C and II.D, trapezoidal shapes have been introduced for the inner stator and outer rotor profiles, in correspondence of the stator slot openings and of the rotor interpolar slots. As a first approximation, these profiles can be interpreted as equipotential surfaces which are perpendicular at any point to the field lines inside the airgap, and thus a main airgap surface can be determined. Thus, it becomes possible to extrapolate a first approximated shape of the functions $r_{G}(\lambda, \alpha)$ and $\varepsilon_{G}(\lambda, \alpha)$ and thus of $\mu_{E}(\lambda, \alpha)$. The unknown variable in such an approach is the field lines geometry. Several techniques have been proposed in literature to determine functions similar to the equivalent function $\mu_{E}(\lambda, \alpha)$ proposed in this paper. Most of these methods are based on magneto-static FE evaluations and neglect the effects of the variation due to the airgap radius $r_{G}(\lambda, \alpha)$ [17], [18], thus taking only the effects of $\varepsilon_{G}(\lambda, \alpha)$ into account. Also, [17] and [18] neglect the damper cage effects on the machine operations. In this work, the initial shape of $\mu_{E}(\lambda, \alpha)$ is defined according to the rationale based on the trapezoidal variation discussed above, while its refinement is supported by a FE test. In particular, the parameters $a, b, e, f, g$ and $i$ shown in Figures 2 and 3 are tuned by direct comparison of the no-load voltage waveforms obtained through the proposed model with those determined via FE. A single transient with motion simulation is thus necessary and the characteristic parameters are modified accordingly within a limited variation range until an acceptable accuracy is achieved in terms of match between analytical and $\mathrm{FE}$ results. This parameter identification process is carried out for one no-load operating point only and the tuned quantities are then used to predict any other no-load and on-load operation of the SG. It is worth mentioning that the choice of using a transient with motion FE simulation is driven by the need of including the damper cage effects on the voltage shape of the studied SG. In this way, the tuned parameters inherently take such effects into account.

To facilitate the understanding of such a tuning procedure and of the overall methodology, a high-level flowchart of the process is illustrated in Fig. 5. Before going into the details of the inductance matrix determination and of all the relevant waveforms, the damper cage modelling approach is described in the next section. The proposed approach represents one of the main novelty of this work, as it has never been presented in the form shown below.

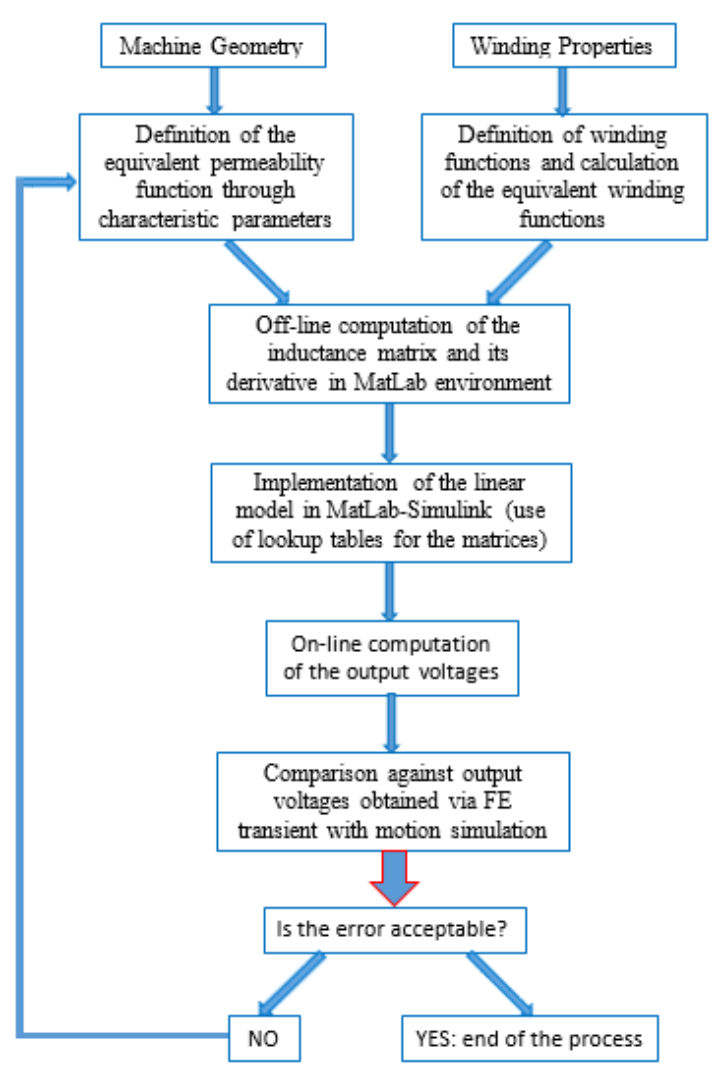

Fig. 5. High-level flow chart of the overall methodology..

\section{Circuital Model of the Damper Cage}

The damper cage modelling approach utilized for achieving the goals of this paper has been initially described in [14], where however no experimental validation is present. Due to the importance of such a model in the overall framework presented here, its main features and implications are recalled in this section.

From a structural point of view, the damper cage can be of complete or incomplete type. In both cases, it consists of longitudinal bars located inside the poles connected through effective low-resistance end paths consisting in ring sectors or press-plates (as in the considered case study). When a complete damper cage is implemented, the adjacent groups of bars are connected through effective low-resistance paths, such as proper end rings similarly to squirrel cages of induction machines. On the other hand, in an incomplete damper cage, the different groups of bars are not connected at all.

Typically, the equivalent circuit topology of complete damper windings is obtained by moving one of the end rings (say the rear one) on the same plane of the other ring. The planar net shown in Fig. 6.a is then achieved, where the straight connections between the inner and outer rings represent the generic $b$ bars of the winding which are usually grouped according to the number of pole pairs $p$ of the machine (for example 2 in Fig. 6.a). In this graph, the number of natural loops is evidently equal to $b+l$ including the central loop. The same approach can be used to obtain the plain net representing incomplete damper cages, as shown in Fig. 6.b for a similar example. In this case the number of natural loops is reduced to 
$b-2 * p$ since $2 * p+1$ loops are suppressed when removing the connections between bars located in adjacent poles. a)

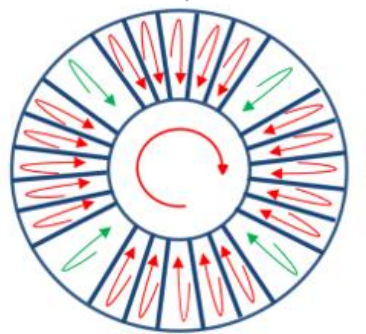

b)

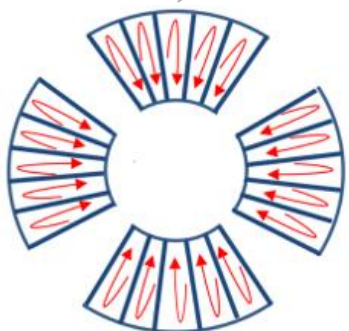

Fig. 6. Equivalent circuit topology of complete (left) and incomplete (right) damper windings.

The loop currents highlighted in Fig. 6 refer to the classical general approach where each equivalent phase comprises two adjacent bars and their end connections belonging to the outer and inner rings. In particular, apart from the inmost ring loop, red arrows identify the intra-pole loops belonging to one specific salient pole, whereas green arrows identify inter-pole loops spanning between adjacent salient poles.

Concerning the applicability of the incomplete cage model, it is worth recalling that in reality the bars and the end connections are not effectively insulated from the rotor magnetic core. However, in several situations, the conductive path between different groups of bars features a much higher resistance with respect to the connections existing between adjacent bars within the same pole. In the considered case study the bars are connected to an aluminium press-plate which is shaped as the profile of the rotor lamination shown in Fig. 1, involving a much longer path. Obviously, a larger resistance in the end connections tends to limit to a smaller value the current flowing in the corresponding loop. In addition, the inter-pole loops span over a much larger tangential portion of the main airgap, but most of it consists of the related inter-pole vane where the thickness of the main airgap is also much larger, meaning that the expected value of flux density is much smaller. Therefore, depending on the location of the lateral bars inside the salient poles, the flux linked to the inter-pole loops is expected to be smaller with respect to intra-pole loops. This will lead to a relatively smaller induced voltage and thus to produce a smaller current, keeping also into account the larger resistances of end connections. Therefore, it turns out that in many practical cases an approximated reduced model neglecting the inter-pole and end-ring loop currents may still provide results whose accuracy could be acceptable depending on the purpose of the model, thus possibly representing an attractive trade-off for accuracy vs. complexity.

The next section presents the conceptual passages which lead to consider "compound" equivalent phases of the damper cage, each composed by $4 p$ bars properly connected throughout the salient poles.

\section{A. The compound representation}

In salient-pole SGs, typically, the bars feature a symmetrical distribution with respect to the pole axis. This is also the case of the considered alternator. Therefore, the physical symmetry related to the number of pole pairs $p$ of the machine can be extended to the cage and also to the set of relevant currents, unless very unusual operating conditions are considered (see Section III.C). In other words, the pattern of the currents flowing in the bars and end-rings inside any pole pair sector can be assumed to be identical in all the other sectors, thus resulting in a reduced number of independent loop currents: $b / p+1$ for complete and $b / p-2$ for incomplete damper windings. In case that a full odd symmetry stands for the electromagnetic aspects, it can be assumed that the current patterns related to the bars located in the 2 adjacent poles are just opposite. In this scenario, the number of independent cage loop currents is then further reduced to $b /(2 * p)+1$ and to $b /(2 * p)-1$ for complete and incomplete cages, respectively.

The observations carried out above permit to obtain an alternative, simplified damper cage equivalent topology that can be embedded in the analytical model. In fact, the equivalent loop phases being considered can be properly connected in series/anti-series throughout the $2 * p$ poles of the machine, as shown in Fig. 7 for an incomplete cage. All of the hypotheses above are verified for the $400 \mathrm{kVA}$ SG used as vehicle to investigate the proposed concepts. Although the end connections between bar groups are realized through the use of press plates, the equivalent paths are much longer than the distance between adjacent bars, also due to the low number of poles the machine is featured with. In some cases, when the machine has a high number of poles and the connections are realized with continuous end rings [2], such an assumption is weak and a complete cage has to be adopted. However, the compound representation can be still applied, as the hypothesis relative to opposite current patterns is verified also for the elementary inter-pole bar loops, where the loop currents shown in green in Fig. 6a flow. In conclusion, the damper cage can be therefore considered as "incomplete" and the number of compound phases used to model it in this new scenario is reduced to 5, whereas an additional equivalent compound loop should be included when a complete cage is adopted. The total number of phases in the machine is then $1+3+5=9$ : this is therefore the dimension of the vector differential equation shown in (2) that has to be solved in order to estimate the voltage waveforms.

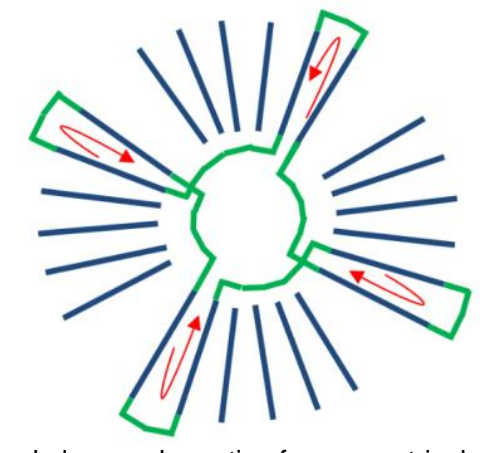

Fig. 7. Compound planar schematic of a symmetrical cage loop

\section{$B$. The winding functions}

Having introduced the concept of the compound representation of the equivalent cage loop phases (see Fig. 7), it is important to show how the relevant WFs are modified with respect to their classical versions (see Fig. 6) and to highlight the implications resulting from this assumption. A comparison between the WFs related to a single cage loop and to the compound phase incorporating the 4 cage loops located in 
corresponding positions along the rotor periphery is reported in Figures $8 \mathrm{a}$ and $8 \mathrm{~b}$, respectively. In its classical version, the EWF calculated according to (4) will be a positive-negative square waveform (as shown in red in Fig. 8a), usually asymmetric, whatever is the trend of the equivalent permeability function. This implies that the product between the EWFs of any couple of cage loops is not null for any value of $\alpha$. In turns, this means that the different loop phases will be magnetically coupled to each other, besides being coupled to the field and armature windings. On the other hand, the WF of any of the compound phase features inherently an odd symmetry periodicity with respect to 1 pole pitch, as shown in Fig. 8b. Considering that the equivalent permeability function of any normal symmetric machine is either constant or features a period just equal to 1 pole pitch, it can be easily concluded that the EWF will result just equal to the basic WF. This means that the product between the EWFs of any couple of compound loops is null for any value of $\alpha$, as they do not share any interval where both are non-zero. This in turns implies that they result not magnetically coupled and the resulting sub-matrix becomes diagonal (i.e. the mutualinductances are all null). This is not achieved even when considering only the set of loops related to one pole by exploiting the assumed symmetry hypotheses, as in [19]. Therefore, one can conclude that by using the compound phases not only the size of the system (2) is minimized, but also the complexity of the inductance matrix is reduced, thus attenuating the computational burden. This represents a highlight of this work, where a balance of accuracy against computational resources is a main objective function.

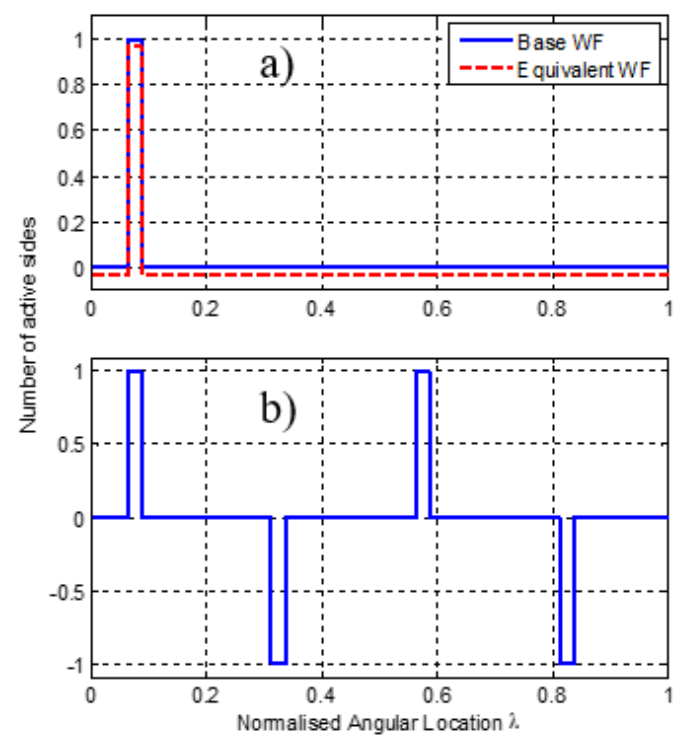

Fig. 8. Winding functions for the damping cage loops of the considered case study: a) classical representation; b) compound representation for symmetrical machines.

\section{Further Considerations}

All the assumptions above stand for machines provided with a symmetrical damper winding and when normal operating conditions are considered. In fact, the hypothesis on the currents featuring opposite waveforms in corresponding bars of adjacent poles is always verified, even in the case of asymmetric load conditions, unless any type of factor comes to compromise the inherent machine symmetries or any event producing an 'asymmetrical' response of the damping bars occurs. These include asymmetrical winding structures (e.g. fractional slot winding, shifted damper cages, etc.), inaccurate manufacturing, defective mounting, internal faults, etc. However, all these events are not taken into consideration in this work, as the main goal is that of evaluating the no-load voltage waveforms of the considered SG. In conclusion, the not-null elements of $\mathbf{L}(\alpha)$ involving the cage loops will be then evaluated by using the EWFs of the type shown in Fig. 8b.

\section{The Hybrid Analytical-Numerical Approach}

In the previous sections, the incremental steps necessary to obtain and correctly implement the equivalent permeability function (see Section II) and the EWFs (see Section III) relative to the SG under analysis are illustrated. These procedures allow to calculate the inductance matrix through the integral given in (1), which is analytically implemented in its full form without assuming sinusoidal approximations as done in [15] and with a purely analytical approach, as opposed to FE ones usually proposed for such purposes [18-20]. The machine inductances are reported in this section as well as the hybrid analyticalnumerical model used to get the output voltage waveforms.

\section{A. The Inductances}

The inductances and their derivatives with respect to the rotor position $\alpha$ are calculated off-line through (1). Their trends vs. $\alpha$ are shown in Figures 9-11 and the following observations can be carried out:

1) In Fig. 9, the self-inductance of the field winding (first subplot), the mutual-inductance between field winding and one of the rotor cage loops (second subplot) and the selfinductance of one cage loop (third subplot) are plotted. It can be noticed that the variation against $\alpha$ of such inductances is just due to the stator slot openings. The integration interval was in fact limited to one stator slot pitch to further reduce the computation effort.

2) In Fig. 10, the stator self- and mutual-inductances are shown. Their dependence on $\alpha$ is due to the anisotropies featured by both the stator and rotor structures. In Fig. 11, the mutual-inductances between the field winding (indicated as FW in the legend) and the 3 stator phases can be observed in the top subplot, while in the bottom one the mutual-inductances between one cage loop (indicated as $\mathrm{CL}$ in the legend) and the 3 stator windings are shown. Also in this case the calculation of (1) was carried out by opportunely limiting the integration interval according to the symmetries featured by the SG.

When the inductance matrix $\mathbf{L}(\alpha)$ is determined as well as the matrices $\partial \mathbf{L}(\alpha) / \partial \alpha$ and $\mathbf{R}$, it becomes possible to solve the system (2). Due to the induced nature of the bar currents, a numerical solution is needed to estimate such quantities. Hence, after being properly manipulated, (2) can be implemented in the Simulink environment as shown in Fig. 12. 


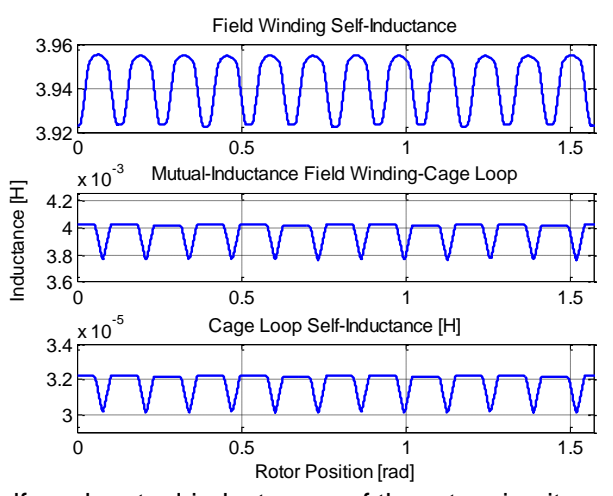

Fig. 9. Self- and mutual-inductances of the rotor circuits.

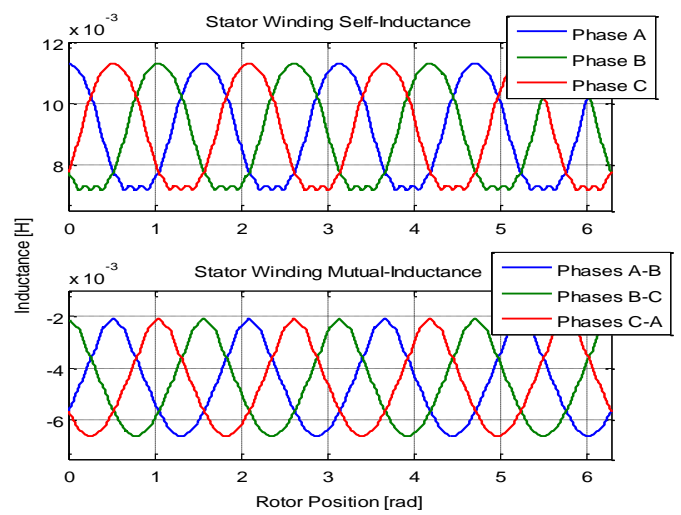

Fig. 10. Stator self- and mutual-inductances.

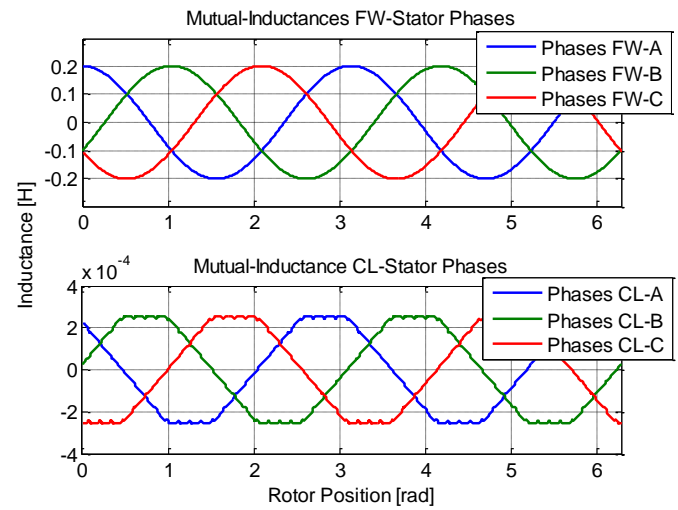

Fig. 11. Mutual-inductances between rotor and stator circuits.

\section{B. The Numerical Solution}

Figure 12 illustrates the schematic of the implemented hybrid approach. The state variables are obviously the currents flowing inside the phases. To simplify the numerical problem resolution and making the model as flexible as possible, an additional voltage drop represented by the diagonal impedance matrix $\mathbf{Z}_{\text {add }}$ is added to system (2). To simulate any operating condition, all the terms contained in $\bar{v}$ are imposed equal to zero except the field winding term, while the terms in $\mathbf{Z}_{\text {add }}$ inherent to the stator phases are set according to the apparent power and power factor that one wants to investigate. For example, the no-load operation can be simulated by imposing a very large resistive value (with null values of the inductive and capacitive parameters, i.e. unity power factor) practically ensuring that the stator currents will be almost null. Considering all this, it is now possible to obtain the no-load and on-load voltage waveforms of the considered SG. The relevant results are reported in the next section, in comparison against the FEand the experimentally-evaluated corresponding waveforms.

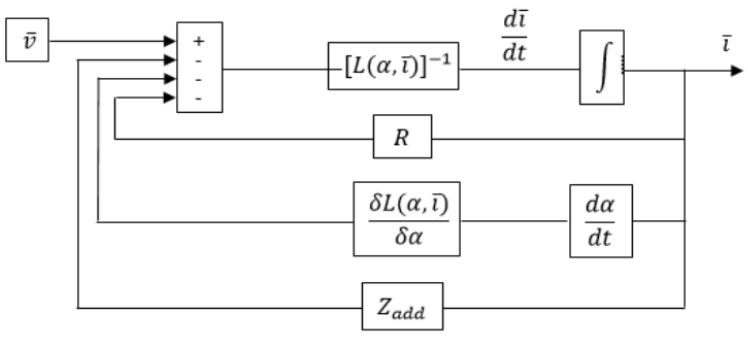

Fig. 12. General linear model of the electrical aspects.

\section{Model Validation Against FE and Experimental RESULTS}

This section is aimed at validating the concepts described alongside the paper against FE and experimental results. First, the major features of the FE model and of the experimental are described. Finally, the validation comparison and the relevant results are provided and discussed in-detail.

\section{A. The FE Model}

A 2-D FE electromagnetic model of the 400-kVA SG was built according to the processes highlighted in [6], [10], [11]. To be consistent with the assumption related to the linear behaviour of the ferromagnetic materials used for the stator and rotor cores, a high permeability magnetic steel is firstly employed for the analysis. A transient with motion evaluation is then performed at no-load operation, with the field winding voltage imposed, the three-phase stator winding open-circuited and the damper bars connected in parallel and modelled as solid conductors to account for the potential non-uniform current distribution over the bars' cross-section.

\section{B. The Experimental Platform}

The experimental platform consists of an induction machine drive, acting as the prime mover, whose speed loops controls the frequency of the considered SG. This is mounted on the same shaft of the induction motor. Given that the no-load voltage prediction represents the main objective of this work, the generator's stator terminals were left open-circuited during the test. The rotor field winding was fed by a DC current provided by a DC power supply through the use of a slip rings and brushes set. A general picture of the machine test bench is shown in Fig. 13. The voltage waveforms were recorded during the test through the use of a typical power analyser. The same general set-up was used for estimating the machine on-load voltage waveforms, with the stator terminals connected to a variable, purely resistive three-phase load bank.

\section{Comparison of Results}

A comparison between the no-load waveforms provided by the analytical and FE methods is shown in Fig. 14, for a value of the excitation current equal to the $29 \%$ of the base field current, having defined the latter as that current value which provides the rated voltage when the armature phases are opencircuited. This value is taken on the linear portion of the no-load characteristic of the considered generator. An excellent 
similarity between the no-load phase voltage waveforms can be observed, proving the validity of the analytical-numerical approach. Additional values of the excitation current are considered in [14], where the results confirm the excellent match between analytical and FE curves.

Although the proposed model is based on the assumption of linear behaviour of the ferromagnetic materials, it is important to observe the deviations of the waveforms obtained through the implemented model from those evaluated by a FE analysis, when in the relevant model non-linear materials are employed. Fig. 15 shows this comparative exercise, where a value equal to the $29 \%$ of the base field current is used. In the same figure, the experimental no-load voltage waveform is also included. A good match can still be observed between the analytically- and FE-evaluated curves. In particular, it can be observed that the hybrid model slightly overestimates the high-frequency harmonics, whereas the main phenomena are accurately predicted. This is also confirmed by the experimental curve, which excellently matches with the FE one.

When different values of the field current are considered on the linear portion of the no-load characteristic, the general trend of the analytical predictions and the relevant errors against FE and experimental results (not shown in this paper for space limitation reasons) are very similar to those observable in Figures 15 and 16. On the other hand, when the rated no-load output voltage is carefully investigated, it becomes clear that the hybrid model presents some limitations. For such conditions the machine operates slightly after the saturation knee and the prediction error increases. In Fig. 17, the comparison between analytical and experimental no-load voltage waveforms is provided, showing good match at the fundamental frequency and some evident discrepancies at higher harmonic components.

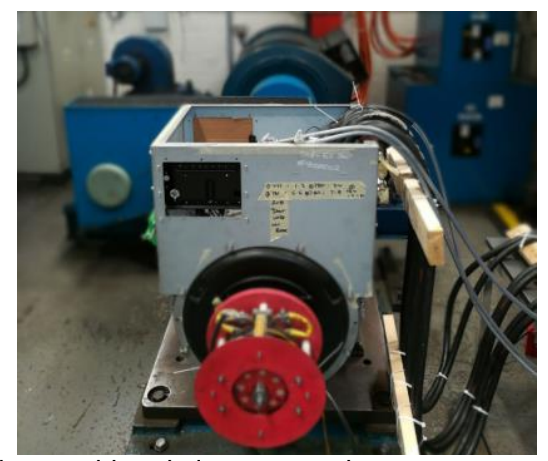

Fig. 13. The considered alternator under test.

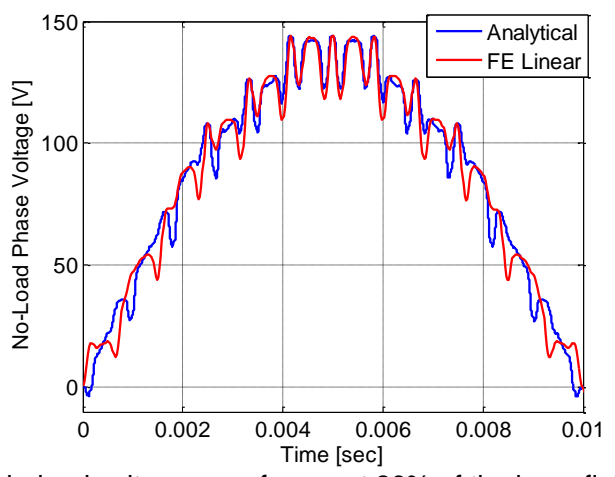

Fig. 14. No-load voltage waveforms: at $29 \%$ of the base field current.

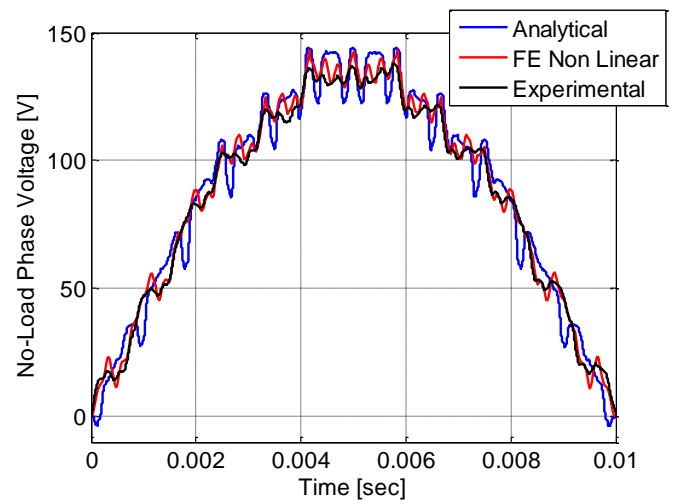

Fig. 15. No-load voltage waveforms at $29 \%$ of the base field current.

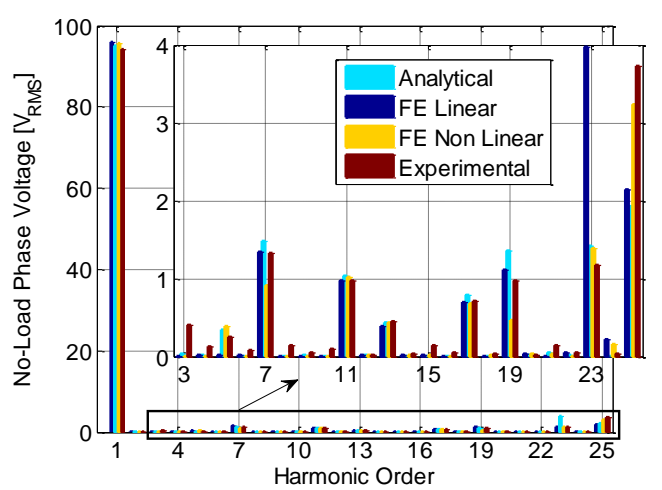

Fig. 16. Harmonic amplitude spectrum of the no-load voltage waveforms: analytical vs. FE with linear materials vs. FE with non-linear materials vs. experimental results.

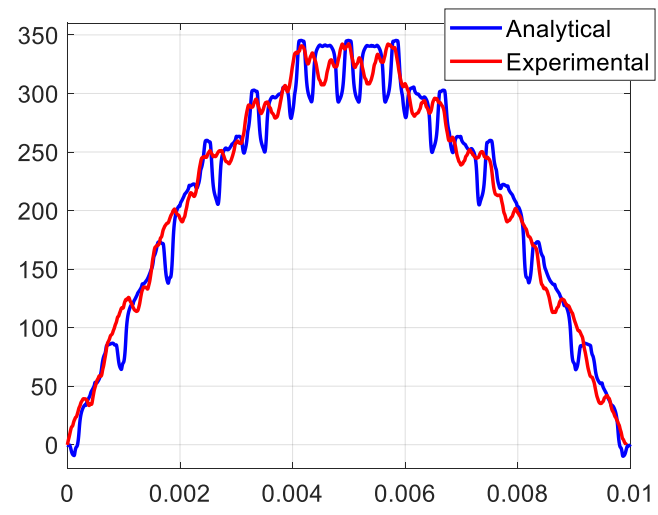

Fig. 17. No-load voltage waveforms at $100 \%$ of the base field current.

To prove the model flexibility and its accuracy also at load operation, the results obtained through the linear hybrid model are compared against experimental measurements carried out at various loadings, at unity power factor and rated output voltage. The comparison is performed in terms of THD, as this represents the most appropriate indicator of the voltage waveforms' quality. Table I summarizes this comparative analysis, confirming that the analytical-numerical predictions slightly overestimate the voltage shape distortion, while achieving an excellent accuracy at the fundamental frequency. In fact, at $50 \mathrm{~Hz}$, both hybrid and experimental results register a voltage value of $400 \mathrm{~V}$ with the same field current. 
TABLE I

VOLTAGE THD AT DIFFERENT LOADINGS AND UNITY POWER FACTOR COMPARISON BETWEEN HYBRID AND EXPERIMENTAL RESULTS

\begin{tabular}{ccc}
\hline \hline \multirow{2}{*}{ Loading } & \multicolumn{2}{c}{ Voltage THD } \\
\cline { 2 - 3 } & Hybrid & Experimental \\
\hline $5 k W$ & $5.75 \%$ & $4.67 \%$ \\
\hline $10 k W$ & $5.75 \%$ & $4.64 \%$ \\
\hline $20 k W$ & $5.72 \%$ & $4.57 \%$ \\
\hline $50 k W$ & $5.68 \%$ & $4.44 \%$ \\
\hline \hline
\end{tabular}

\section{Discussion}

The results presented above prove the validity of the proposed model, especially when linear operating conditions are assumed (see Fig. 14). On the other hand, some limitations are observed under non-linear conditions, although the similarity between results was still however very good (see Figures 15 and 17). In Fig. 16, the harmonic amplitude spectra of the waveforms shown in Figures 14 and 15 are illustrated. In particular, the analytical results are compared against linear FE, non-linear FE and experimental ones. The figure shows up the $25^{\text {th }}$ harmonic, which belongs to the first group of slotting harmonics centred around the $24^{\text {th }}$ spectral component. Fig. 16 confirms that the hybrid model can accurately predict the noload voltage waveforms of salient-pole SGs, showing small inaccuracies just in terms of high-frequency harmonics (zoomed in in the figure). To overcome these challenges, the most representative non-linear parts of the machine may be included as done in [16] and [18], where relatively complex FEand reluctance-based approaches were used, resulting in increased complexity and computation times which are against the objectives of this paper. Considering this, it is clear that the proposed model unavoidably lacks the capability to represent completely all of the details, as some aspects such as saturation do affect the machine operation. However, the main scope of this paper is to propose a method able to describe and capture the most important aspects of the SG operation with a low computational burden (when compared to more accurate and detailed approaches), while still ensuring an acceptable accuracy level. In fact, for the considered case study the hybrid model solves in about $20 \mathrm{sec}$ against $1 \mathrm{~h}$ and $15 \mathrm{~min}$ employed by the non-linear FE model implemented for the purposes of this paper

Besides the comparison against purely numerical methods, it is important to highlight the benefits of the damper cage modelling technique presented in this work with respect to those classically used by machine designers. In Table II, it can be observed that if the considered SG was modelled via the classical general method (first row), the system order would be about 4 times higher than in the proposed technique (third row). The classical model requires a much larger number of off-line calculations to describe the inductances and 19 more currents have to be calculated during on-line resolution. If the reduced classical model (e.g. [19], second row) was used, the system order would become equal to that of the proposed technique. However, all the mutual inductances between cage loops should be determined and taken into account, as they would be not null as in the model presented in this paper. All this highlights that much faster solutions can be obtained thanks to the implementation of the compound equivalent phases described in Section III, while keeping the same level of accuracy in comparison with the classical techniques. All these computational benefits highlighted for a specific case study become even more enhanced when SGs featuring high number of poles and bars-per-pole (e.g. large hydrogenerators) are investigated, provided that an odd-symmetric structure is present. It is also worth mentioning that if a design optimization (e.g. involving the optimal repositioning of the bars over one pole [6], [21]) was to be performed via the proposed method, the positive impact on the computational times would be also augmented with respect to classical methods, given that very high numbers of individuals are usually investigated in such studies to find optimal solutions.

TABLE II

BENEFITS OF THE PROPOSED VS. CLASSICAL MODEL FOR A CASE STUDY

\begin{tabular}{ccccc}
\hline \hline Models & $\begin{array}{c}\text { Analysed } \\
\text { portion }\end{array}$ & $\begin{array}{c}\text { System } \\
\text { order }\end{array}$ & Implications & Complexity \\
\hline \multirow{2}{*}{$\begin{array}{c}\text { Classical } \\
\text { literature }\end{array}$} & $\begin{array}{c}\text { Whole } \\
\text { machine }\end{array}$ & 28 & $\begin{array}{c}\text { Not-null CL mutual } \\
\text { inductances }\end{array}$ & High \\
\cline { 2 - 5 } & $\begin{array}{c}\text { One pole } \\
\text { pitch }\end{array}$ & 9 & $\begin{array}{c}\text { Not-null CL mutual } \\
\text { inductances }\end{array}$ & Medium \\
\hline $\begin{array}{c}\text { Proposed } \\
\text { model }\end{array}$ & $\begin{array}{c}\text { Whole } \\
\text { Machine }\end{array}$ & 9 & $\begin{array}{c}\text { Null CL mutual } \\
\text { inductances }\end{array}$ & Low \\
\hline \hline
\end{tabular}

\section{CONCLUSIONS}

This paper aims at accurately estimating the voltage waveforms in a particular salient-pole SG, when operating in linear conditions, by implementing a mixed analyticalnumerical model that solves much faster than the classic FE method. The model was successfully validated against FE and experimental results.

Focus was given to the damper cage, whose modelling was carried out in an alternative way compared to the classical literature. This allowed to drastically reduce the number of equations to be solved. In fact, the considerations carried out in Section III allowed to model the entire generator without compromising the order of the system of equations to be solved. On the contrary, due to the fact that the whole machine is modelled, it became possible to obtain null values of all the mutual inductances between the cage loops. This last aspect represents a further significant step with respect to the state-ofthe-art, as it allows to appreciably reduce the complexity of the overall system.

All the benefits highlighted throughout the paper resulting from the application of the proposed methods can have a significant impact in terms of solution speeds, especially when very large numbers of machines are investigated to find out optimal design solutions. Therefore, it can be concluded that the proposed model can be used as a better and improved version of the classical ones even for design purposes, potentially resulting in faster optimization processes which are nowadays very much required and implemented in both academic and industrial environments. 


\section{REFERENCES}

[1] D. Fallows, S. Nuzzo, A. Costabeber, M. Galea, "Harmonic reduction methods for electrical generation: a review," IET Generation, Transmission \& Distribution vol. 12, no. 13, pp. 3107-3113, 2018.

[2] A. Tessarolo, C. Bassi, D. Giulivo, "Time-stepping finite-element analysis of a 14-MVA salient-pole shipboard for different damper winding design solution," IEEE Trans. Ind. Electron., vol. 59, no. 6, pp. 2524-2535, Jun. 2012.

[3] S. Nuzzo, M. Galea, C. Gerada, N. Brown, "Analysis, modelling and design considerations for the excitation systems of synchronous generators," IEEE Transactions on Industrial. Electronics, vol. 65, no. 4, pp. 2996-3007, Apr. 2018.

[4] V. Madonna, P. Giangrande, M. Galea, "Electrical power generation in aircraft: review, challenges and opportunities", IEEE Trans. on Transportation Electrification., vol. XX, pp. 1-1, 2018.

[5] G. Traxler-Samek, T. Lugand, A. Schwery, "Additional losses in the damper winding of large hydrogenerators at open-circuit and load conditions", IEEE Trans. Ind. Electron., vol. 57, pp. 154-160, Jan. 2010.

[6] S. Nuzzo, M. Degano, M. Galea, C. Gerada, D. Gerada, N. L. Brown, "Improved damper cage design for salient-pole synchronous generators," IEEE Transactions on Industrial. Electronics, vol. 64, pp. 1958-1970, 2017.

[7] E. Kimbark, Power System Stability-Volume 1. Hoboken, NJ, USA: Wiley-IEEE Press, Chapter XIV, 1995.

[8] H. Karmaker, A.M. Knight, "Investigation and simulation of fields in large salient-pole synchronous machines with skewed stator slots," IEEE Trans. Energy Convers., vol. 20, no. 3, pp. 604-610, Sep. 2005.

[9] A.M. Knight, H. Karmaker, K. Weeber, "Use of a permeance model to predict force harmonic components and damper winding effects in salient-pole synchronous machines," IEEE Trans. Energy Convers., vol. 17, no. 4, pp. 709-716, Dec. 2002.

[10] S. Nuzzo, M. Galea, C. Gerada, N. Brown, "A fast method for modelling skew and its effects in salient-pole synchronous generators", IEEE Trans. Ind. Electron., vol. 64, no. 10, pp. 7679-7688, 2017.

[11] S. Nuzzo, P. Bolognesi, G. Vakil, D. Fallows, C. Gerada, N. Brown, M. Galea, "A methodology to remove Stator Skew in Small-Medium Size Synchronous Generators via innovative damper cage designs", IEEE Transactions on Industrial. Electronics, Early Access, 2018.

[12] E. Wallin, M. Ranlof, U. Lundin, "Design and construction of a synchronous generator test set-up," presented at the Int. Conf. Electrical Machines (ICEM), Rome, Italy, Sep. 6-8, 2010, Paper RF-008982.

[13] G. Traxler-Samek, T. Zickermann, A. Schwery, "Cooling airflow, losses and temperatures in large air-cooled synchronous generators", IEEE Trans. Ind. Electron., vol. 57, no. 1, pp. 172-180, Jan. 2010.

[14] S. Nuzzo, P. Bolognesi, M. Galea, and C. Gerada, "A hybrid analyticalnumerical approach for the analysis of salient-pole synchronous generators with a symmetrical damper cage," in IEEE International Electric Machines and Drives Conference (IEMDC), USA, 2017.

[15] P. Bolognesi, "A mid-complexity analysis of long-drum-type electric machines suitable for circuital modelling," Proc. of ICEM 2008 Conf., Vilamoura, Sep. 2008.

[16] R. Wang, S. Pekarek, M. L. Bash, A. Larson and R. V. Maaren, "Incorporating Dynamics in a Mesh-Based Magnetic Equivalent Circuit Model of Synchronous Machines," IEEE Transactions on Energy Conversion, vol. 30, no. 3, pp. 821-832, Sept. 2015.

[17] A. Tessarolo, "Accurate Computation of Multiphase Synchronous Machine Inductances Based on Winding Function Theory," IEEE Trans. on Energy Conversion, vol. 27, no. 4, pp. 895-904, Dec. 2012.

[18] S. Hemmati, S. S. Kojoori, S. Saied and T. A. Lipo, "Modelling and experimental validation of internal short-circuit fault in salient-pole synchronous machines using numerical gap function including stator and rotor core saturation," IET Electric Power Applications, vol. 7, no. 5, pp. 391-399, May 2013.

[19] S. Keller, Mai Tu Xuan and J. J. Simond, "Computation of the no-load voltage waveform of laminated salient-pole synchronous generators," IEEE Trans. Ind. Applic.., vol. 42, no. 3, pp. 681-687, May-June 2006.

[20] A. Z. Gbégbé, B. Rouached, J. Cros, M. Bergeron and P. Viarouge, "Damper Currents Simulation of Large Hydro-Generator Using the Combination of FEM and Coupled Circuits Models," IEEE Transactions on Energy Conversion, vol. 32, no. 4, pp. 1273-1283, Dec. 2017.

[21] S. Nuzzo, P. Bolognesi, G. Vakil, D. Fallows, C. Gerada, N. L. Brown, M. Galea, "A methodology to remove Stator Skew in Small-Medium Size Synchronous Generators via innovative damper cage designs," DOI
10.1109/TIE.2018.2864699, IEEE Transactions on Industrial Electronics, 2018.

\section{BIOGRAPHIES}

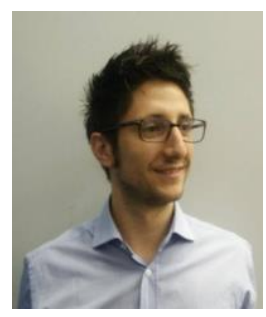

Stefano Nuzzo received the B.Sc. and M.Sc. degrees in electrical engineering from the University of Pisa, Pisa, Italy, in 2011 and 2014, respectively.

He received his Ph.D. degree in Electrical Machine Design in 2018 from the University of Nottingham, Nottingham, U.K, where he is currently working as a Research Fellow within the Power Electronics, Machines and Control (PEMC) Group. His current research interests are the analysis, modelling and optimizations of electrical machines, with focus on salient-pole synchronous generators and brushless excitation systems for industrial power generation applications.

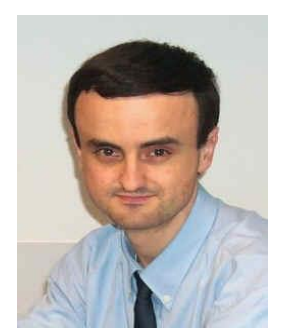

Paolo Bolognesi (M'99) received the M.Sc. and Ph.D. degrees in Electrical Engineering from the University of Pisa, Italy, respectively in 1995 and 1999.

He joined then the University of Pisa as a post-doc junior researcher and finally as a Senior Researcher and Appointed Professor in 2001. His research interests focus on modelling, analysis and design of electromagnetic devices, including unconventional electrical machines and electromechanical actuators, as well as on innovative topologies and modulation/control techniques for static converters, also for applications related to electric and hybrid vehicles.

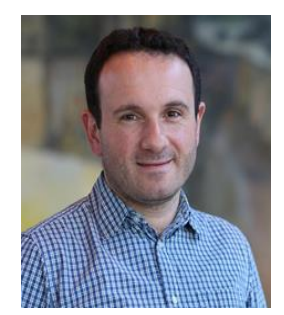

Chris Gerada (M'05-SM'12) received the Ph.D. degree in numerical modeling of electrical machines from The University of Nottingham, Nottingham, U.K., in 2005.

He was a Researcher with The University of Nottingham, working on high-performance electrical drives and on the design and modeling of electromagnetic actuators for aerospace applications. Since 2006, he has been the Project Manager of the GE Aviation Strategic Partnership. In 2008, he became a Lecturer in electrical machines, in 2011, as an Associate Professor, and in 2013, a Professor at The University of Nottingham. His main research interests include the design and modeling of high-performance electric drives and machines.

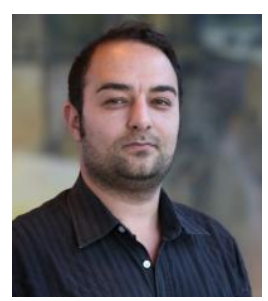

Michael Galea (M'13-SM'18) received the Ph.D. degree in electrical machines design from the University of Nottingham, Nottingham, U.K. He was a Research Fellow with University of Nottingham. He is currently the Head of School of Aerospace, University of Nottingham Ningbo China, where he is also the Director of Aerospace. He currently lectures in Electrical Drives and in Aerospace Systems Integration and manages a number of diverse projects and programmes related to the more/all electric aircraft, electrified propulsion, and associated fields. His main research interests include design, analysis, and thermal management of electrical machines and drives (classical and unconventional), the more electric aircraft and electrified and hybrid propulsion. Dr. Galea is a Fellow of the Royal Aeronautical Society. 